\title{
Interaction of Acoustic Disturbances with Micro-Cavities for Ultrasonic Absorptive Coatings
}

\author{
Guillaume A. Brès* and Tim Colonius ${ }^{\dagger}$ \\ California Institute of Technology, Pasadena, CA 91125, U.S.A. \\ Alexander V. Fedorov ${ }^{\ddagger}$ \\ Moscow Institute of Physics and Technology, Zhukovsky, Moscow region, 140180, Russia
}

\begin{abstract}
Numerical simulations are performed to investigate the interaction of acoustic waves with an array of equally-spaced two-dimensional micro-cavities on an otherwise flat plate without external boundary-layer flow. This acoustic scattering problem is important in the design of ultrasonic absorptive coatings (UAC) for hypersonic laminar flow control. The reflection coefficient, characterizing the ratio of the reflected wave amplitude to the incident wave amplitude, is computed as a function of the acoustic wave frequency and angle of incidence, for coatings of different porosity, at various acoustic Reynolds numbers relevant to hypersonic flight. Overall, the numerical results validate predictions from existing theoretical modeling. In general, the amplitude of the reflection coefficient has local minima at some specific frequencies. A simple model to predict these frequencies is presented. The simulations also highlight the presence of resonant acoustic modes caused by coupling of small-scale scattered waves near the UAC surface. Finally, the cavity depth and the porosity are identified as the most important parameters for UAC design. Guidelines for the choice of these parameters are suggested.
\end{abstract}

\section{Nomenclature}

$\begin{array}{ll}A r & \text { Cavity aspect ratio } \\ a & \text { Speed of sound } \\ b & \text { Cavity half-width } \\ \tilde{C} & \text { Dynamic compressibility } \\ c & \text { Phase speed } \\ c_{p} & \text { Specific heat at constant pressure } \\ f & \text { Frequency } \\ H & \text { Cavity depth } \\ k & \text { Wave number } \\ m & \text { Propagation constant } \\ P & \text { Pressure } \\ p & \text { Acoustic pressure } \\ p_{i} & \text { Initial pulse amplitude } \\ P r & \text { Prandtl number } \\ \mathcal{R} & \text { Reflection coefficient } \\ R e & \text { Acoustic Reynolds number } \\ s & \text { Cavity spacing } \\ T & \text { Temperature } \\ t & \text { Time }\end{array}$

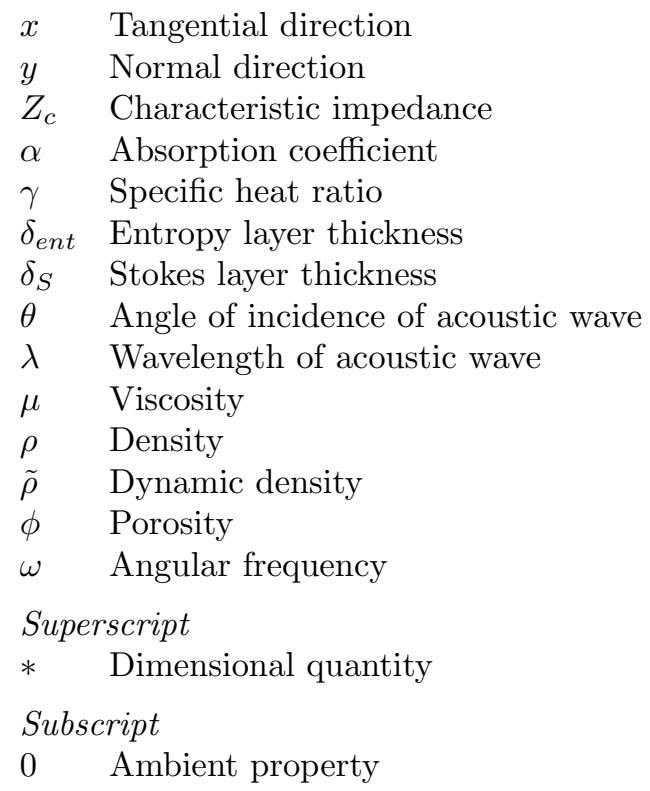

*Postdoctorate Fellow, Dept. of Mechanical Engineering, Member AIAA

$\dagger$ Professor, Dept. of Mechanical Engineering, Member AIAA

$\ddagger$ Professor, Dept. of Aeromechanics and Flight Engineering, Member AIAA 


\section{Introduction and previous work}

\section{A. Motivations}

Hypersonic laminar flow control for delayed laminar-turbulent transition is an important component of economically viable hypersonic vehicles. ${ }^{1,2}$ Wind tunnel experiments and theoretical studies ${ }^{3-8}$ have already demonstrated that an ultrasonically absorptive coating (UAC), which consists of a thin micro-porous layer, can suppress the second-mode instability and thereby delay transition of predominately two-dimensional boundary layer. However, design and robust implementation of UAC on hypersonic vehicles hinges on the development of accurate and efficient low-order models of the UAC as a boundary condition to traditional computational fluid dynamic and instability codes.

The research thus seeks to validate and improve existing porous-wall boundary-condition models. This motivates us to conduct direct numerical simulations (DNS) for the boundary layer disturbances including unsteady processes within the micro-cavities that constitute the UAC. In particular, we will examine endeffects at the mouths and bottoms of cavities, and clarify dependencies of the UAC performance on the cavity spacing and depth.

In this paper we use DNS to investigate the interaction of incident acoustic waves with an array of equallyspaced micro-cavities on a flat plate surface without flow. Although the external boundary-layer flow is an important component of the UAC modeling, this unit problem is of particular interest, since mechanisms of reflection and absorption of incoming boundary-layer disturbances occur near the cavity edges and inside cavities where the external flow is relatively slow and seems to play a minor role. Furthermore, the acoustic properties of UAC samples should be estimated in an economical way before testing in hypersonic wind tunnels. This can be done using benchmark (no-flow) measurements of the reflection of acoustic waves from the porous coating. Theory and modeling are needed to design, implement and interpret such benchmark experiments.

\section{B. Theoretical model}

We consider the reflection of plane monochromatic ultrasonic acoustic waves by a plane surface covered by a porous coating (figure 1). The coating has a regular structure consisting of equally-spaced slots (2-D cavities). It is assumed that the UAC is absolutely rigid and its temperature is uniform and constant. It is also assumed that: (a) the cavity half-width $b$ and spacing $s$ satisfy the condition $b \sim s \ll \lambda$, where $\lambda$ is the wavelength of incident acoustic wave; (b) the cavity depth $H \sim \lambda$. With these assumptions, the reflection coefficient (complex quantity characterizing the ratio of the reflected wave amplitude to the incident wave amplitude) is given by the relationship ${ }^{9,10}$

$$
\mathcal{R}=\frac{Z_{c} \cos \theta-1}{Z_{c} \cos \theta+1}, \quad Z_{c}=\frac{Z_{c}^{*}}{\rho_{0} a_{0}}=\frac{1}{\rho_{0} a_{0} \phi} \sqrt{\frac{\tilde{\rho}}{\tilde{C}}} \operatorname{coth}(m H), \quad m=i \omega^{*} \sqrt{\tilde{\rho} \tilde{C}} .
$$

Here $Z_{c}$ is the normalized characteristic impedance and $m$ is the propagation constant of the porous layer, $\phi=2 b / s$ is the porosity, $\rho_{0}$ and $a_{0}$ are the density and speed of sound in ambient undisturbed gas, and $\omega^{*}=2 \pi f^{*}$ is the angular frequency of the acoustic wave. The dynamic density $\tilde{\rho}$, the dynamic compressibility $\tilde{C}$, and the propagation constant $m$, are calculated for an isolated deep cavity $(H \gg b)$ using the analytical solution derived by Kozlov et al. ${ }^{11}$ For the case of zero bulk viscosity and infinitesimal Knudsen number (ratio of the molecular mean free pass to the cavity half-width), this solution gives

$$
\tilde{\rho}=\frac{\rho_{0} \Lambda}{\Lambda-\tan \Lambda}, \quad \tilde{C}=\frac{1}{\gamma P_{0}}\left[1+(\gamma-1) \frac{\tan \tilde{\Lambda}}{\tilde{\Lambda}}\right]
$$

where $\Lambda=\sqrt{-i \omega^{*} \rho_{0} b^{2} / \mu_{0}}$ is proportional to the ratio of cavity half-width to the Stokes-layer thickness $\delta_{S}^{*}=\sqrt{2 \mu_{0} / \omega^{*} \rho_{0}}$. Here $\tilde{\Lambda}=\sqrt{\operatorname{Pr}} \Lambda, \mu_{0}$ is the gas viscosity, $\gamma$ is the specific heat ratio, and $\operatorname{Pr}$ is the Prandtl number.

Note that this model accounts for viscous dissipation and heat conductivity inside individual cavities. However, end effects associated with scattering of incoming acoustic waves by the cavity mouth and local effects on the cavity bottom are ignored. Small-scale disturbances, which are generated near the UAC surface, are not considered. There is no coupling between disturbances in neighboring cavities, which may 


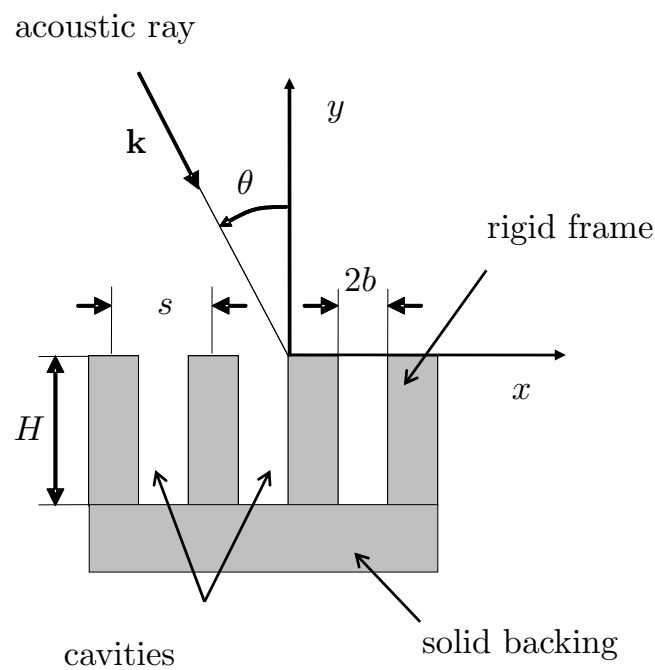

Figure 1. Schematic of the reflection of acoustic wave from equally spaced 2-D cavities

not be true for closely spaced cavities. This motivates us to perform DNS to clarify the aforementioned effects and validate the robustness of the theoretical model by comparisons with DNS solutions.

\section{Direct Numerical Simulations}

\section{A. Numerical methods}

Brès and Colonius ${ }^{12}$ developed an algorithm to solve the full compressible Navier-Stokes (NS) equations and study the flow over three-dimensional open cavities. The equations are solved for all the scales of the flow without the use of any turbulence modeling. The code was modified to model the flow in individual and array of equally-spaced cavities in a rigid surface under a transitional boundary layer. We assume shock-free flow and use sixth-order accurate compact finite difference schemes for streamwise and normal directions, and Fourier-spectral differentiation for homogeneous directions (when present). The usual compressible formulation is used to nondimensionalize the NS equations, where the superscript $*$ refers to the dimensional quantity, and the subscript 0 denotes the ambient undisturbed property:

$$
\rho=\frac{\rho^{*}}{\rho_{0}}, \quad P=\frac{P^{*}}{\rho_{0} a_{0}^{2}}, \quad T=\frac{T^{*} c_{p}}{a_{0}^{2}}, \quad u_{i}=\frac{u_{i}^{*}}{a_{0}}, \quad x_{i}=\frac{x_{i}^{*}}{H}, \quad t=\frac{t^{*} a_{0}}{H} .
$$

The fluid is a perfect gas with specific heat at constant pressure $c_{p}$, Prandtl number $\operatorname{Pr}=0.72$ and $\gamma=1.4$. The temperature at the walls is assumed uniform and constant $\left(T_{w}=T_{0}\right)$. The code can handle any type of block geometry (including the porous surface configuration shown in figure 1) and is fully parallelized using Message-Passing Interface (MPI).

The current study focuses on the acoustic scattering by an array of equally-spaced 2-D micro-cavities without external flow. The acoustic pressure fields $p=P-P_{0}$ generated by an initial acoustic disturbance (subscript $i c$ ) and its reflection (subscript ref) on a solid wall (without cavities) and on a porous wall (with cavities) are computed, and can be decomposed as:

$$
\begin{aligned}
p_{\text {solid }}(x, y, t) & =p_{i c}(x, y, t)+p_{\text {ref,solid }}(x, y, t), \\
p_{\text {porous }}(x, y, t) & =p_{i c}(x, y, t)+p_{\text {ref,porous }}(x, y, t) .
\end{aligned}
$$

We set the amplitude sufficiently small such that the resulting interaction is linear. Assuming that there is no overlapping of the initial and reflected waves, we can then identify the reflected signals at any fixed 
point $\left(x_{0}, y_{0}\right)$, and expand them into Fourier integrals:

$$
\begin{aligned}
p_{\text {ref }, \text { solid }}(t) & =\frac{1}{\sqrt{2 \pi}} \int_{-\infty}^{+\infty} \hat{p}_{\text {ref,solid }}(\omega) e^{-i \omega t} d \omega, \\
p_{\text {ref,porous }}(t) & =\frac{1}{\sqrt{2 \pi}} \int_{-\infty}^{+\infty} \hat{p}_{\text {ref,porous }}(\omega) e^{-i \omega t} d \omega,
\end{aligned}
$$

where $\omega=\omega^{*} H / a_{0}$. Since reflection from a solid wall has a reflection coefficient of one, we can calculate the complex reflection coefficient at $\left(x_{0}, y_{0}\right)$ (i.e., at angle $\left.\theta_{0}\right)$ for the porous wall $\mathcal{R}_{D N S}(\omega)=$ $\hat{p}_{\text {ref,porous }}(\omega) / \hat{p}_{\text {ref,solid }}(\omega)$ and compare it to the theoretical value $\mathcal{R}(\omega)$ in Eq. 1 .

\section{B. Computational setup}

The configuration considered in this study corresponds to 2-D micro-cavities of constant length to depth ratio $A r=2 b / H=0.12$. This value matches the aspect ratio of the cylindrical cavities used in the experiment by Rasheed et al. ${ }^{4}$

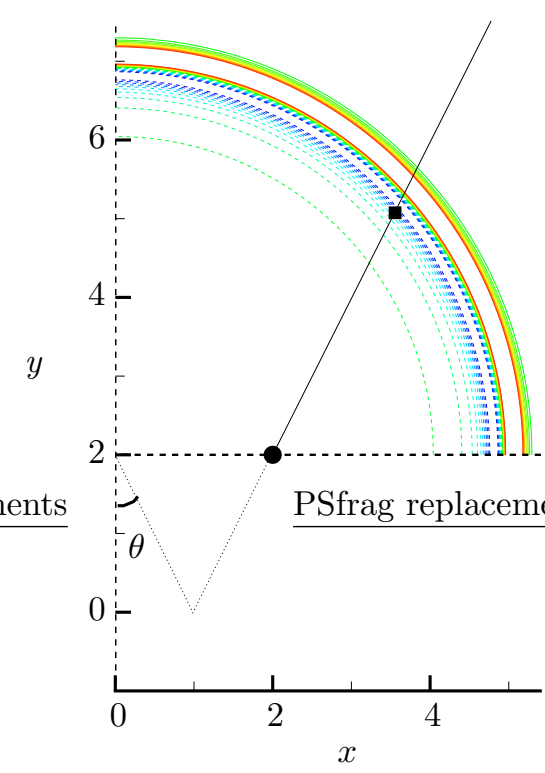

(a)

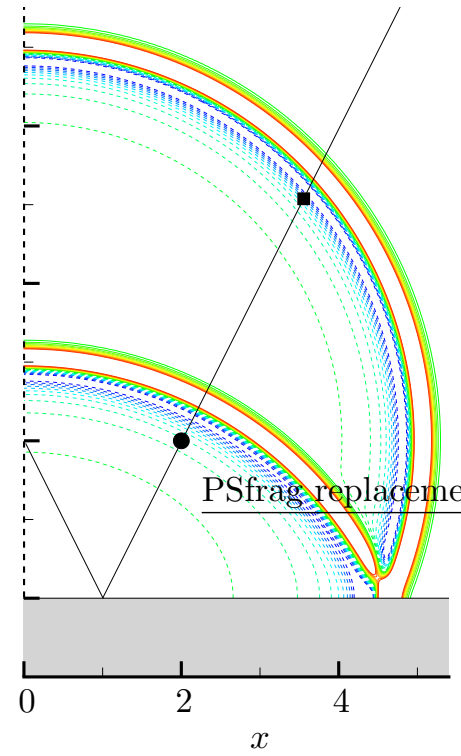

$(b)$

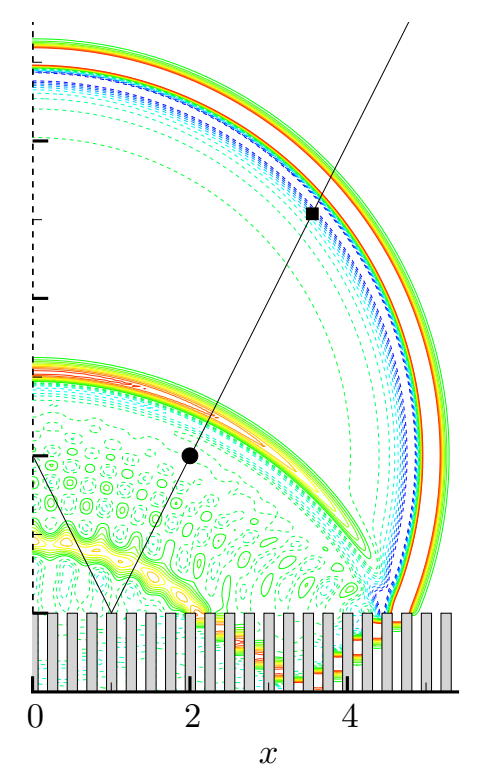

$(c)$

Figure 2. Acoustic pressure field at time $t=10$ for simulations at $R e=100$ without wall (a), with solid wall (b), and with coating of porosity $\phi=0.48(c)$. Twenty contours are shown between $p / p_{i}=-0.01$ and 0.01 , with negative contours dashed. While the maximum pressure of the incoming pulse is $p / p_{i}=0.025$, the contours were chosen to highlight the smaller amplitude reflected waves. The initial acoustic disturbance is located at $(0,2)$. For each angle $\theta$, the acoustic pressure field is recorded at $2 H(\bullet), 5 H(\boldsymbol{m})$, and $10 H$ above the wall. Only a small portion of the computational domain is shown here, with symmetric boundary conditions represented by the black dashed line (---- ).

In practice, the pressure field is measured at multiple locations (for a given height above the wall) to account for the dependence on $\theta$, the angle of incidence of the acoustic wave, and at different heights (i.e., at $2 H, 5 H$, and $10 H$ ) to quantify near-field effects (see figure 2). For all the simulations, the initial disturbance at $t=0$ is a circular acoustic pulse:

$$
p(x, y, 0)=\rho(x, y, 0)-\rho_{0}=p_{i} \exp \left[-\left(\left(x-x_{c}\right)^{2}+\left(y-y_{c}\right)^{2}\right) / \sigma^{2}\right], \quad u(x, y, 0)=v(x, y, 0)=0
$$

with $p_{i}=0.001$ and $\sigma=0.1$. To capture all the successive reflected waves from the porous surface, the simulation are performed up to time $t=40$, until all the disturbances in the flow have died away. To minimize the computational domain, the pulse is located at $\left(x_{c}, y_{c}\right)=(0,2)$ with symmetric boundary conditions at 
$x=x_{c}$. The grid extends up to $30 H$ and $18 H$ in the $x$ - and $y$-direction, respectively, with a large buffer zone at the top and right boundary, to avoid spurious reflections. For the configuration with the porous wall, the mesh contains about one and a half million grid points, with 12 and 100 points across each cavity length and depth, respectively.

Also, to ensure that the reflected waves are measured independently of the initial wave, an additional simulation without the wall (using symmetric boundary conditions at $y=y_{c}$ ) is performed to determine $p_{i c}(x, y, t)$. This particular procedure and choice of initial condition (rather than plane monochromatic acoustic waves) enables the computation of a complete mapping of the reflection coefficient $\mathcal{R}_{D N S}(\theta, \omega)$ in only three simulations (i.e., without wall, with solid wall, and with porous coating), as shown in figure 2 .

\section{Validation}

All the numerical simulations are performed on the same stretched Cartesian grid, with clustering of points near the walls to accurately capture the flow inside and around the pores. In particular, the interaction of acoustic waves with the solid wall leads to the formation of a Stokes layer and entropy layer on the wall surface. Their respective thicknesses can be estimated ${ }^{13}$ as $\delta_{S}=\delta_{S}^{*} / H=\sqrt{A r /(\omega R e)}$, and $\delta_{\text {ent }}=\delta_{S} / \sqrt{\operatorname{Pr}}$, where $R e=\rho_{0} a_{0} b / \mu_{0}$ is the acoustic Reynolds numbers based on the cavity half-width. These features are fully resolved on the chosen computational mesh, except for high Reynolds number (e.g., $R e=1000)$. However, for disturbances of ultrasonic frequency relevant to this study, these lengths are much smaller than the corresponding acoustic wavelength $\lambda=a_{0} / f^{*}$, and the Stokes and entropy layers can therefore be neglected. To validate this assumption and the numerical setup, we also considered a planar pulse at normal incidence:

$$
p(x, y, 0)=\rho(x, y, 0)-\rho_{0}=p_{i} \exp \left[-\left(y-y_{c}\right)^{2} / \sigma^{2}\right]=-v(x, y, 0), \quad u(x, y, 0)=0 .
$$

This configuration corresponds to $\theta=0^{\circ}$, and allows us to limit the computation domain to a single pore with periodic boundary conditions in the $x$-direction. For this case, the grid resolution in each direction is increased by a factor of five. The reflected signal is measured at $1 H, 2 H, 5 H$, and $10 H$ above the wall, and there is no overlapping of the initial and reflected waves in this setup.

First, the reflected signal from the DNS is compared to theory, ${ }^{9}$ using the classical absorption coefficient $\alpha_{c}$ and phase speed $c_{c}$. For the reflected planar pulse traveling in the $+y$ direction, the Fourier coefficients of the pressure signal at $y=y_{1}+\Delta y$ should theoretically satisfy $\hat{p}_{r e f}(y, \omega)=\hat{p}_{r e f}\left(y_{1}, \omega\right) \exp \left(-\alpha_{c} \Delta y\right) \exp \left(-i \omega \Delta y / c_{c}\right)$. Given a pressure time-history at $y=y_{1}$ from the DNS, the theoretical pressure time-history at some distance $\Delta y$ can therefore be reconstructed using Eq. 4. As shown in figure 3, there is excellent agreement between DNS and theory, which validate the numerical resolution of the viscous and thermal spatial absorption, and of the dispersion of the acoustic waves during propagation.

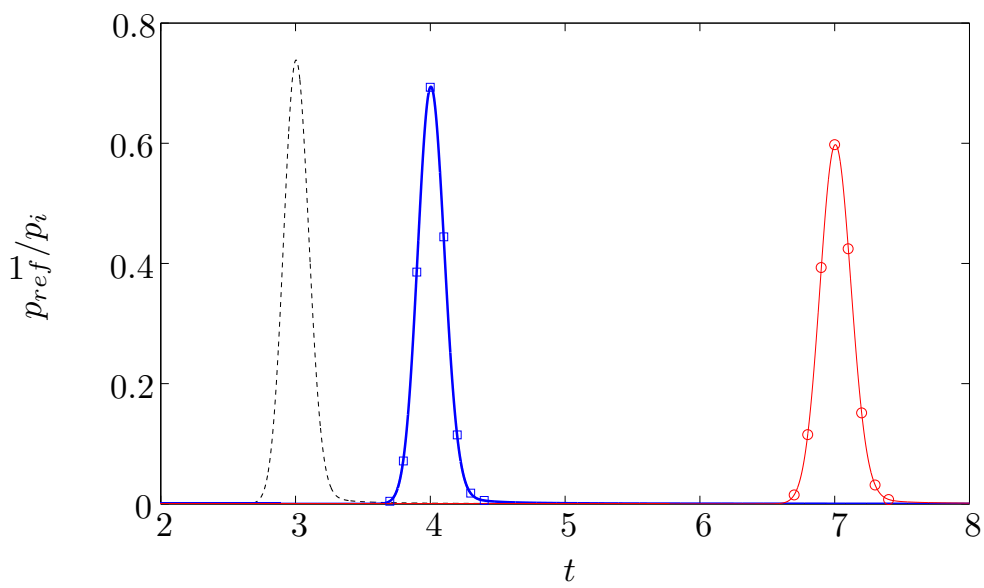

Figure 3. Absorption and dispersion of the planar pulse reflected from the solid surface at $R e=100$ : Pressure time-history at $y=y_{1}=1 H$ from DNS ( - - - ); Pressure time-history at $y=2 H$ from DNS ( -$)$ and from theory $(-\square-)$; Pressure time-history at $y=5 H$ from DNS $(-)$ ) and from theory $(-\bigcirc-)$. 


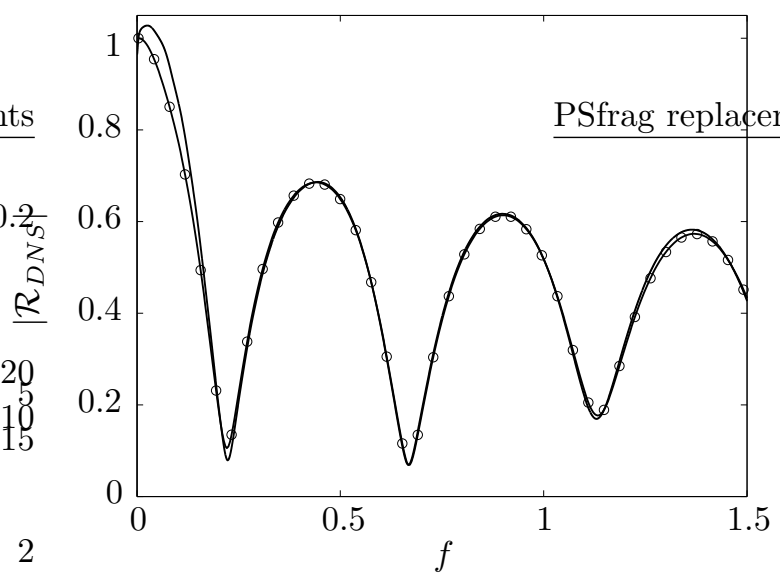

$(a)$

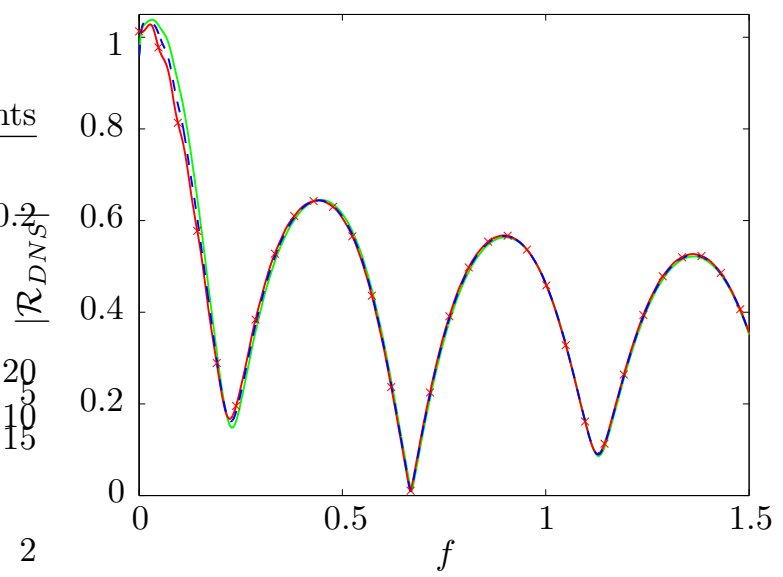

$(b)$

Figure 4. Reflection coefficient for different numerical setup at $R e=100$. (a) Effect of grid resolution and initial condition for $\theta=0^{\circ}$ : circular pulse ( - ), and planar pulse on fine mesh $(\circ)$ ) (b) Effect of height of measurement for $\theta=30^{\circ}$ and circular pulse: at $2 H(-), 5 H(---)$, and $10 H(\times)$ above the wall.

Next, the simulations for the planar pulse on the fine mesh and for the circular pulse on the original mesh are compared for the different Reynolds number considered in this study. Both the Stokes and entropy layers are fully resolved on the fine mesh. The results in figure $4(a)$ show that the reflection coefficients are nearly identical, independent of the grid resolution and initial disturbance. The small discrepancies observed at low frequencies are likely due to the curvature of the wave front in the case of the circular pulse.

Additionally, the reflection coefficients measured at $2 \mathrm{H}, 5 \mathrm{H}$, and $10 \mathrm{H}$ above the surface are compared in figure $4(b)$, for the circular pulse disturbance, at $\theta=30^{\circ}$ and $R e=100$, with $\phi=0.48$. The results show that near-field effects are negligible and that the reflection coefficient is independent of the height of the measurements. The same conclusion is obtained for all angle of incidence, Reynolds number, and porosity. In the remainder of the paper, the results will be presented for measurements at $2 H$.

Different widths, $\sigma$, of the acoustic pulse are also considered, and lead to similar results for the reflection coefficient, up to a frequency $f=f^{*} H / a_{0} \approx 2$. For typical UAC parameters, this range of frequencies corresponds to the ultrasonic frequency band, and is sufficient to capture the frequency of the most amplified second-mode instability waves observed in experiments ${ }^{4,14}$ and numerical simulations. ${ }^{3,15,16}$

\section{Results and discussion}

\section{A. Parametric study}

The numerical simulations are performed at Reynolds numbers $R e=10,100$, and 1000, for coatings of porosity $\phi=0.2,0.48$, and 0.8 . These setup and flow conditions are chosen to enable future comparisons with ongoing experiments ${ }^{17}$ on UAC samples. These values of $R e$ also correspond to the range of acoustics Reynolds numbers relevant for practical UAC (e.g., $b \approx 5$ to $100 \mu \mathrm{m}$ ) in high-altitude (e.g., $30 \mathrm{~km}$ ) hypersonic flight.

\section{Effect of angle of incidence}

Figure 5 shows the complete mapping of the reflection coefficient obtained for a coating of porosity $\phi=0.48$, at $R e=100$. This plot is representative of the results for the UAC of different porosities considered, and $R e \geq 100$. The reflection coefficient shows strong frequency modulations, largely independent of the angle of incidence, here up to $\theta \approx 60^{\circ}$. As $\theta$ increases, an angle is reached where the reflection coefficient is at its minimum value. This behavior is typical of the reflection of acoustic waves obliquely incident on a normallyreacting surface (see figure 6.6.1 in Ref. 9), where the transmitted wave is refracted so that it propagates effectively only perpendicular to the surface (i.e., inside the pores in our case). As $\theta \rightarrow 90^{\circ}$, the theoretical 


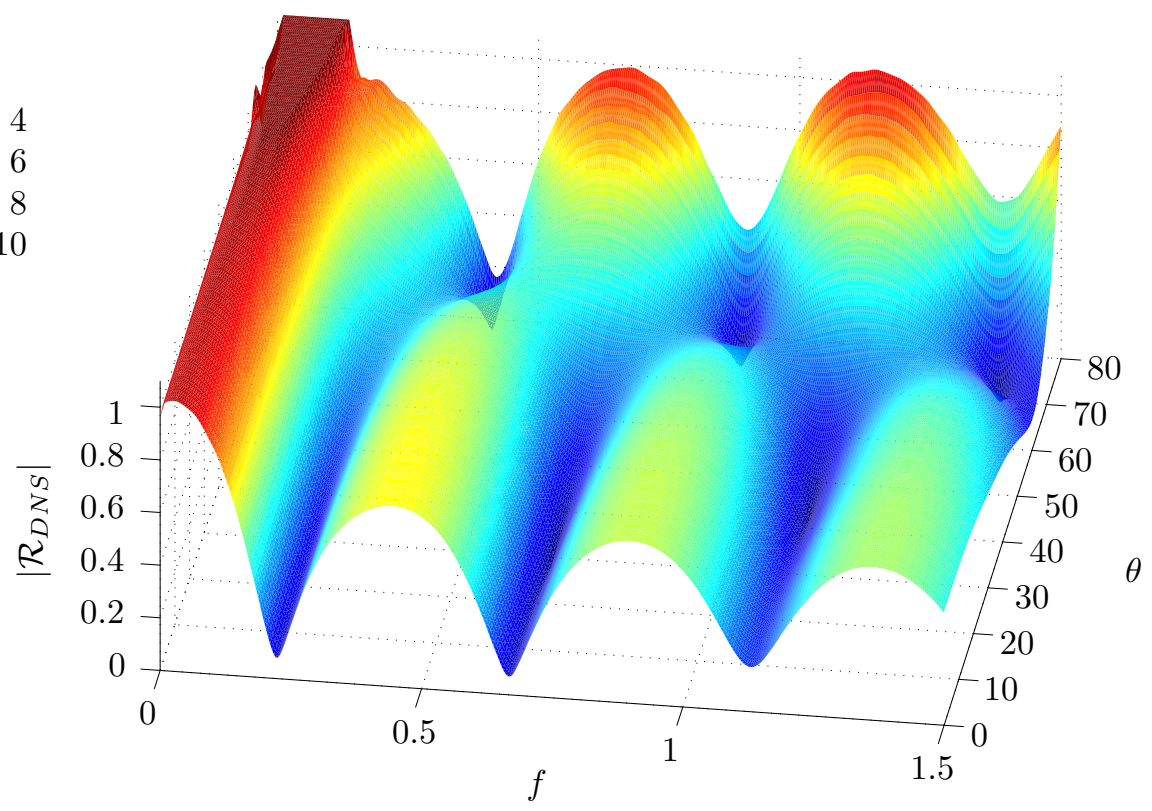

Figure 5. Reflection coefficient amplitude $\left|\mathcal{R}_{D N S}\right|$ as a function of frequency $f$ and angle of incidence $\theta$ for a coating of porosity $\phi=0.48$ at $R e=100$.

reflection coefficient approaches $\mathcal{R}=-1$, and in this limit, the coatings acts as a pressure-release surface. This result is confirmed by the DNS measurements, as shown in figure 6. At high angle of incidence, the ratio of peak pressure amplitude from UAC to solid wall is approximately $90 \%$ (i.e., peaks at $t \approx 23$ in figure $6(b))$, and the waves are in opposition of phase, such that the acoustic pressure goes zero at the top surface in the limit of $\theta=90^{\circ}$.

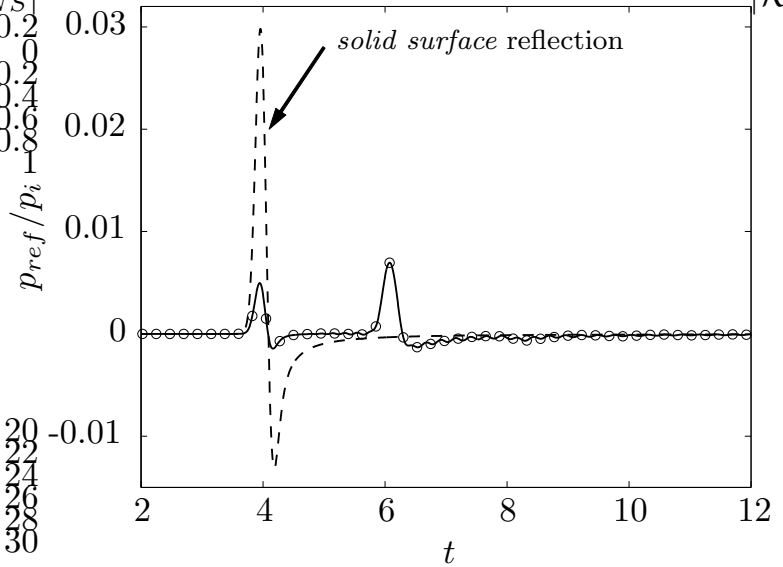

(a)

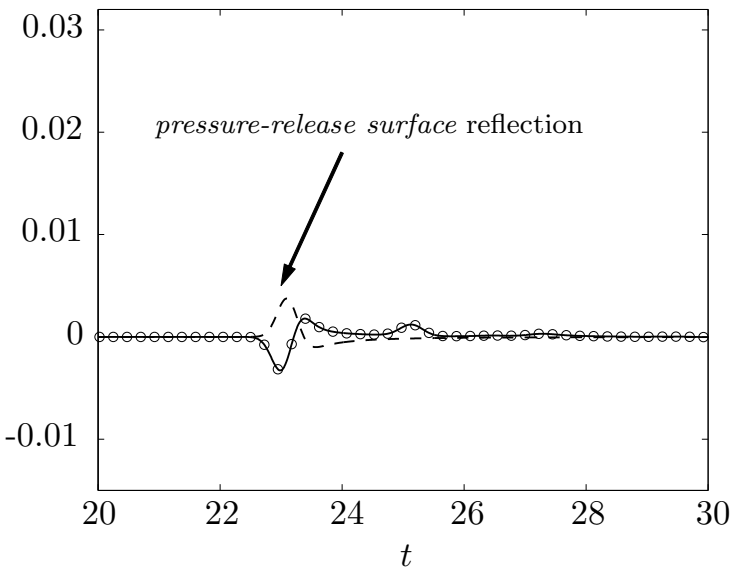

$(b)$

Figure 6. Pressure time-history at $2 H$ above the top surface and $R e=100$ for the solid wall ( ----$)$ and UAC of porosity $\phi=0.8(\circ)$. ( a) Angle of incidence $\theta=0^{\circ}$. (b) $\theta=80^{\circ}$.

For second-mode instability waves, the angle of incidence can be estimated using a WKB approximation. ${ }^{18}$ In the inviscid limit, the pressure amplitude of the second mode is a solution of an eigenvalue problem (Eqs. (5-7) in Ref. 18), and is expressed as a superposition of incident and reflected acoustic waves. The angle of 
incidence $\theta_{\text {inc }}$ (measured from the $x$-axis) is estimated as

$$
\tan \theta_{i n c}=\lambda_{r}(0) / \alpha_{r} \approx \sqrt{\frac{M_{e}^{2}(U(0)-c)^{2}}{T(0)}-1}=\sqrt{\frac{M_{e}^{2} c^{2}}{T_{w}}-1},
$$

where $c=c^{*} / U_{e}$ is the phase velocity, $T_{w}=T_{w}^{*} / T_{e}^{*}$ is the wall temperature, and $M_{e}=U_{e} / a_{e}$ is the local Mach number. Here, the subscript $e$ denotes quantities at the upper boundary-layer edge. For the second mode at $M_{e} \geq 6$, the phase velocity is $c \approx 0.9$. For cold-wall conditions relevant to experiment in shock tunnels, ${ }^{4,19}$ the wall temperature is $T_{w} \approx 1$, which gives at high Mach number $\theta_{\text {inc }} \approx 81^{\circ}-87^{\circ}$, close to $90^{\circ}$ (i.e., normal incidence). For moderate cooling ${ }^{8}$ and adiabatic wall, ${ }^{5}$ the estimated angle of incidence decreases to $\theta_{i n c} \approx 74^{\circ}$ and $64^{\circ}$, respectively. In all cases, the angle of incidence relevant for UAC design is smaller than $\theta=90^{\circ}-\theta_{i n c}=36^{\circ}$. Therefore, our analysis will mainly focus on the reflection coefficient at normal incidence.

\section{Effect of Viscosity}

Figure 7(a) shows the time-history of the reflected signal $p_{r e f} / p_{i}$ at normal incidence for a solid wall and a porous surface $(\phi=0.48)$, at the different Reynolds numbers considered (Re $=10,100$, and 1000). The main reflections from the top solid wall, and from the bottom of the cavity can be identified at time $t \approx 4$ and $t \approx 6$, respectively. The corresponding reflection coefficient is shown in figure $7(b)$.

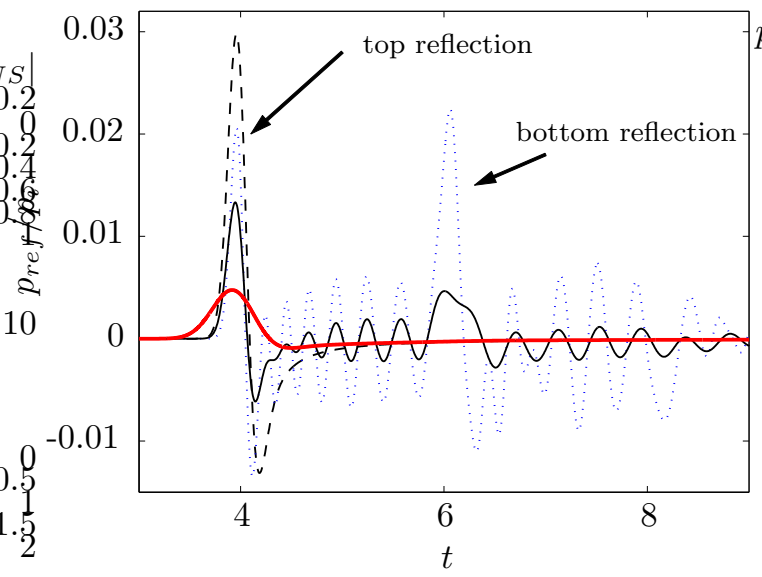

$(a)$

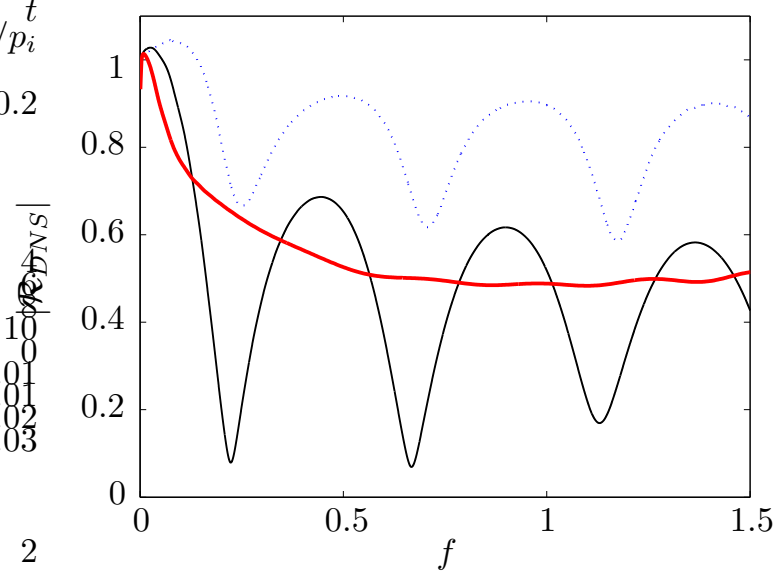

(b)

Figure 7. Effect of viscosity on the reflection of acoustic wave from a coating of porosity $\phi=0.48$ at normal incidence $\left(\theta=0^{\circ}\right): R e=10(-), R e=100(-)$, and $R e=1000(\cdots \cdots)$ ). ( $(-$ ) Pressure time-history at $2 \mathrm{H}$ above the top surface. For comparison, the reflected signal from the solid wall at $R e=100$ is also presented $(----)$. (b) Reflection coefficient amplitude.

At higher Reynolds numbers $(R e=100$ and 1000), reflections from the bottom of the cavities lead to destructive/constructive reinforcement at some specific frequencies. These frequencies, corresponding to local minima and maxima of the reflection coefficient, are only weakly dependent on the Reynolds number. As viscosity is increased (e.g., $R e=10$ ), there is no additional reflection from the cavity bottom because of the increase in dissipation inside the pores, and the reflection coefficient monotonically decreases with frequency.

As mentioned in section IIC, we suspect that the curvature of the wave front introduced some discrepancies at low frequencies, and our method overestimates the reflection coefficient for $f<0.1$. However, this limitation does not affect the range of ultrasonic frequency relevant for UAC design. Also, the additional pressure oscillations observed at late times in particular at $R e=1000$ and 100 correspond to the resonant modes discussed in section D.

The influence of the viscosity can therefore be summarized qualitatively as follow: there is a critical Reynolds number above which acoustic disturbances are not completely absorbed inside the pores. In that case, interference between incoming and outgoing (reflected from the cavity bottom) waves leads to 
significant decrease of the reflection coefficient at specific frequencies. A simple model for the prediction of these frequencies is presented in section C.

\section{Effect of porosity}

To investigate the influence of porosity, simulations are performed for $\phi=0.2,0.48$, and 0.8 , keeping the same cavity aspect ratio. The pressure time-history and reflection coefficient at normal incidence for $R e=100$ are shown in figure $8(a)$ and $(b)$, respectively. As expected, the amplitude of the reflection from the top surface (i.e., the peak at $t \approx 4$ in figure $8(a)$ ) decreases with porosity, while the reflection from the bottom of the cavity (i.e., the peak at $t \approx 6$ ) increases. The frequencies corresponding to local minima and maxima of the reflection coefficient are largely unaffected by the change in porosity. In contrast, the amplitude of the reflection coefficient increases as porosity decreases. This result is consistent with the limit value $|\mathcal{R}|=1$ for $\phi=0$, and with the observations that UAC performance increases with porosity. The porosity is therefore a critical parameter for the scattering and absorption of acoustic waves by porous surface, and this feature is further discussed in section E.

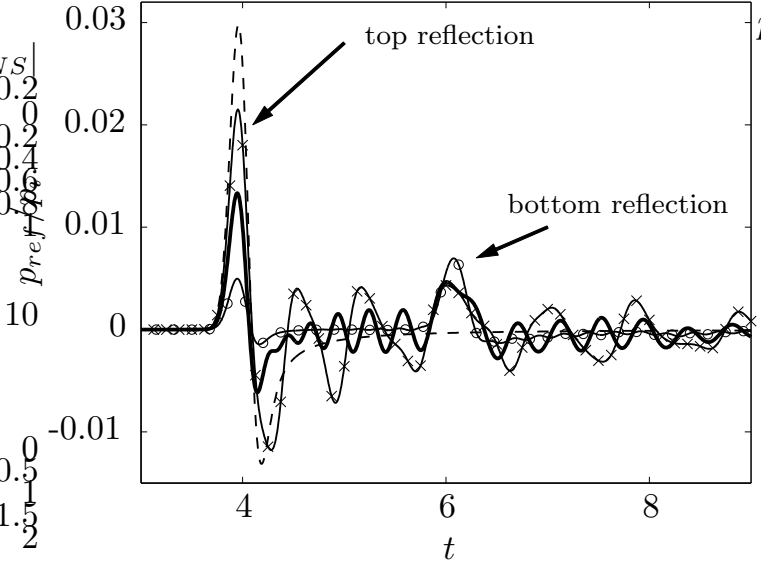

(a)

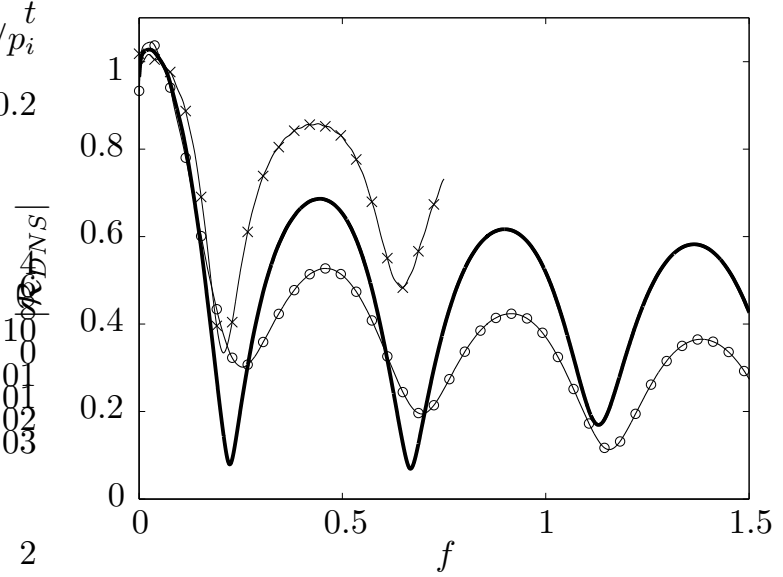

(b)

Figure 8. Effect of porosity on the reflection of acoustic wave from a porous surfaces at $R e=100$ and normal incidence $\left(\theta=0^{\circ}\right)$ : porosity $\phi=0.2(\times), \phi=0.48(-)$, and $\phi=0.8(\circ)$. (a) Pressure time-history at $2 H$ above the top surface. For comparison, the reflected signal from the solid wall is also presented $(----)$. (b) Reflection coefficient amplitude

As mentioned previously, the additional oscillations observed in the pressure time-history correspond to a resonant mode. From figure $8(a)$, it is clear that the oscillation frequency and amplitude depend on porosity. In particular, for $\phi=0.2$, the presence of the resonant mode of frequency approximately 0.83 strongly affects the calculation of the reflection coefficient, and the data are not reliable for $f \geq 0.8$. Therefore, the results are not shown in figure $8(b)$. The resonant mode is examined in more detail in section D.

\section{B. Comparison of DNS with theory}

The comparisons between the reflection coefficient obtained from DNS and from theory at $R e=100$ are presented in figure $9(a),(b)$, and $(c)$, for porosity $\phi=0.8, \phi=0.48$, and $\phi=0.2$, respectively. Overall, there is good agreement between the DNS results and the theoretical predictions. The formulation described in section IB accurately captures the dependence of the reflected acoustic waves on the angle of incidence, frequency and porosity. As expected, the Stokes and entropy layers on the cavity mouth and bottom, which are resolved in the DNS but not accounted for in the theoretical model, have little influence on the reflection of ultrasonic acoustic waves.

In particular, the theory confirms that the reflection coefficient is approximately independent of the angle of incidence for $\theta \leq 30^{\circ}-35^{\circ}$. There is also good agreement on the specific frequencies of minimum reflection coefficient, for both low and high angle of incidence. The pressure time-history at $\theta=0^{\circ}$ and $\theta=80^{\circ}$ in figure 6 gives some insight on the dependence of these frequencies on the angle of incidence. At normal 


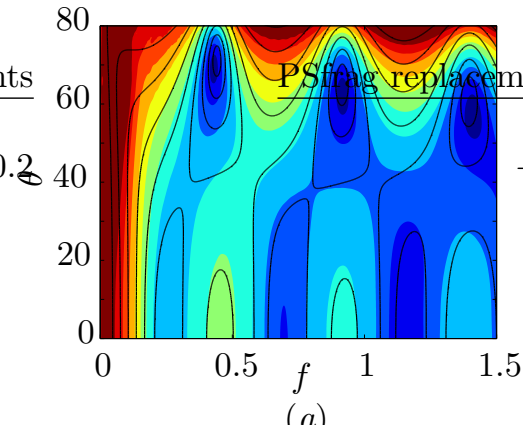

(a)

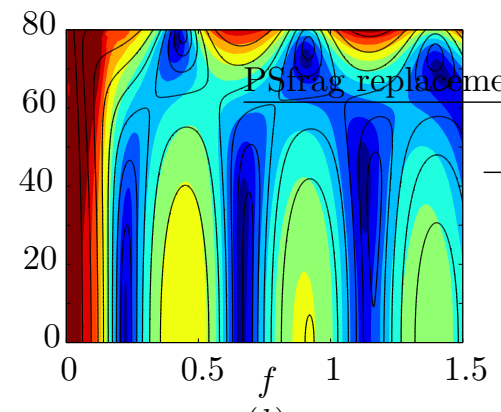

(b)

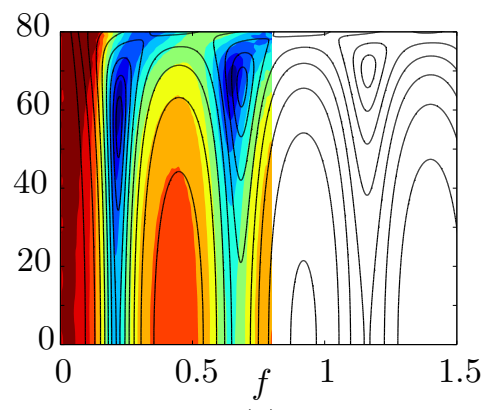

$(c)$

Figure 9. Comparison of the reflection coefficient amplitude from DNS (flooded contours) and theory ( - ) at $R e=100:(a)$ porosity $\phi=0.8 ;(b) \phi=0.48 ;(c) \phi=0.2$. Ten contours are shown between $|\mathcal{R}|=0$ and 1 .

incidence in figure 6(a), the reflected wave from the top surface of the porous wall is in phase with the reflected wave from the solid surface, but approximately in opposition of phase at $\theta=80^{\circ}$ in figure $6(b)$. This observation suggests that the specific values of the frequencies of minimum reflection coefficient are related to the phase between the reflected waves, which is the starting point of the analysis presented in section C.

Detailed comparison between theory and DNS results at normal incidence (using the planar pulse on the fine mesh) is shown in figure 10. For all the porosities and Reynolds number considered in this study, the theoretical predictions match the DNS measurements well, in particular at low frequency. The agreement is good even for high porosity, where neighboring pores are closely spaced and the theory neglects the coupling of disturbances. The discrepancy increases with frequency, most likely because the theory assumes that the acoustic wavelength $\lambda$ is much larger than the cavity half-width $b$ and the spacing $s$. For instance, for $f=1.5$ and porosity $\phi=0.48$, the ratio $s / \lambda \approx 0.4$. Also, for lower Reynolds number (i.e., $R e=10$ ), the viscous effects lead to significant absorption and dispersion of the incoming wave. In this case, a smaller initial pulse width $\sigma$ may be required to fully capture the reflection coefficient at high frequency and better match the theoretical predictions.

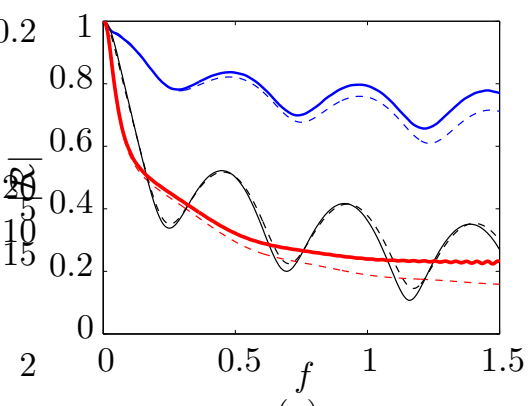

$(a)$

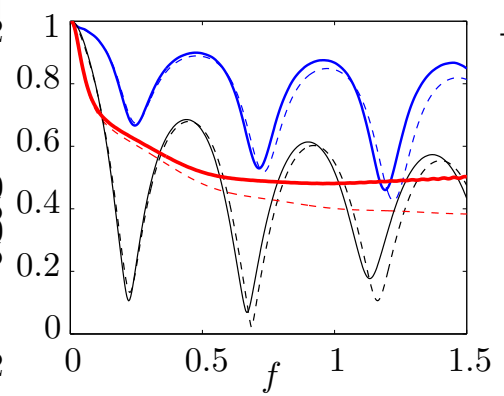

(b)

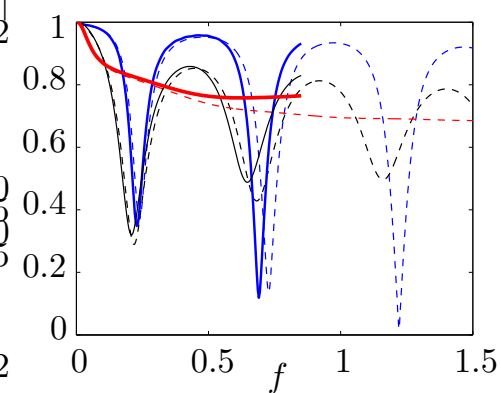

(c)

Figure 10. Comparison of the reflection coefficient at normal incidence from DNS (solid line) and theory (dashed line) at Reynolds number $R e=10$ ( $~$ ) , $R e=100$ ( $\square$ ), and $R e=1000$ ( — ). (a) Porosity $\phi=0.8 ;(b) \phi=0.48 ;(c) \phi=0.2$.

Note that for the low porosity case in figure $9(c)$ and $10(c)$, the DNS results are affected by the resonant mode for $f>0.85$ and are not shown. This resonant mode is not captured in the theoretical model because it originates from small-scale disturbances generated near the UAC surface and coupling between neighboring cavities, as discussed in section D. 


\section{Estimate of the specific frequencies of minimum reflection}

We present here a simple model to predict the frequencies of minimum reflection coefficient. For a plane monochromatic wave traveling in the $+y$ direction (i.e., reflected wave from the solid wall), the solution to the wave equation is of the form $p_{1} \exp [i(\omega t-k y)]$. Similarly, the reflected wave from the porous surface is of the form $p_{2} \exp [i(\omega t-k(y+\Delta y))]$, where $\Delta y=2 H$ correspond to the distance traveled by the wave inside the pores. Minima of the reflection coefficient are obtained when the reflection from the solid wall and the porous surface are in opposition of phase, that is $k_{r} \Delta y=(2 n-1) \pi$, where $k_{r}$ is the real part of the wave number $k=\omega^{*} / a_{0}$, and $n=1,2,3$, etc. In the inviscid approximation, the wave number in dimensional form is $k_{r}=k=2 \pi f^{*} / a_{0}$, and the frequencies of minimum reflection coefficient relevant for UAC are estimated as:

$$
\frac{f^{*} H}{a_{0}}=f_{n}=(2 n-1) / 4,
$$

where $n=1,2$, and 3 lead to the specific frequencies $f_{1}=0.25, f_{2}=0.75$, and $f_{3}=1.25$, respectively.

The effect of viscosity can also be estimated using the absorption coefficient $\alpha_{w}^{*}$ for wall losses in wide pipes. ${ }^{9}$ This approximation is valid for pores wide compare to the Stokes layer thickness (i.e., $b / \delta_{S}^{*} \gg 1$, which is approximately the case in our study for $R e \geq 100$. The presence of the viscous boundary layer modifies the wave speed $c_{w}^{*}$ of the acoustic wave such that $c_{w}=c_{w}^{*} / a_{0} \approx 1-\alpha_{w}^{*} / k$. In that case, $k_{r}=\omega^{*} / c_{w}^{*}=k / c_{w}$, and the frequencies of minimum reflection coefficient are now the solution of the equation

$$
\frac{f_{n}}{c_{w}\left(f_{n}\right)}=(2 n-1) / 4 \quad n=1,2,3 .
$$

The predicted frequencies for $R e=100,1000$, and the inviscid limit are compared to DNS and theory in figure 11. The results are presented for the different porosities considered, which lead to some scattering of the data. Overall, there is a reasonable agreement between the estimations and the measured frequencies of minimum reflection, with approximately less than $10 \%$ error. The formulation with viscosity effects accurately captures the Reynolds number dependence of these frequencies, namely that they decrease with viscosity. While the prediction at $R e=1000$ provides good result, the decrease in frequency at $R e=100$ is overestimated, most likely because the assumption that $b / \delta_{S}^{*} \gg 1$ starts to break down.

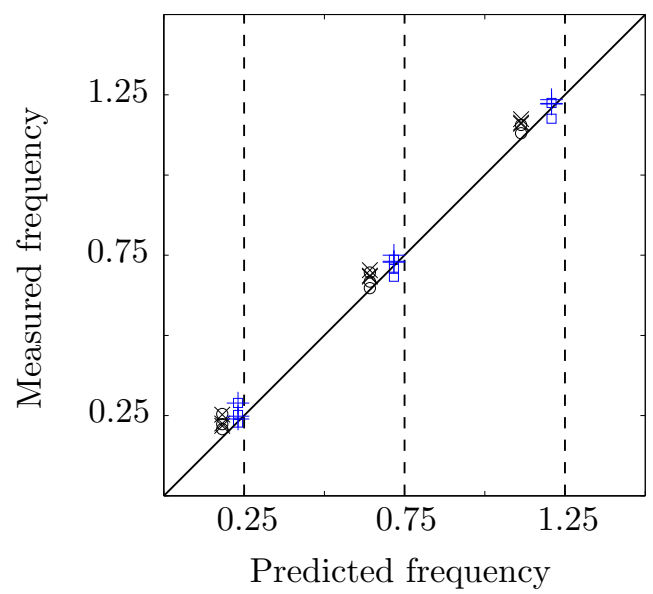

Figure 11. Comparison of the frequency predicted from Eq. 7 with frequencies of minimum reflection measured from DNS ( $\circ R e=100 ; \square R e=1000)$ and theory ( $\times R e=100 ;+R e=1000)$. The inviscid estimate from Eq. 6 is shown by the dashed line $(----)$.

This model is based on the assumption that the phase difference between the reflected waves is introduced only by the traveled distance in the pores. As mentioned in section A1, an additional phase shift is observed at high angle of incidence. In the inviscid approximation, if the waves are initially in opposition of phase (as in figure $6(b)$ ), then the frequencies of minimum reflection coefficient are now $f=n / 2, n=1,2$, and 3. These estimates are in good agreement with the DNS and theoretical results for $\theta>70^{\circ}$ in figure $9(a)$ and $(b)$. Also note that a similar condition can be derived to predict the frequencies of maximum reflection coefficient. 


\section{Resonant modes}

In most of our simulations, we observe the presence of additional oscillations in the flow field, with a frequency strongly dependent on porosity. We argue here that these oscillations correspond to resonant modes caused by small-scale disturbances generated near the UAC surface and coupling between neighboring cavities. In this context, the term "resonant" is used to qualify the mode frequencies in a broad sense, even though the oscillations are actually slowly damped and decay in time.

In this mechanism, the interaction of the acoustic wave with the porous surface generates scattered waves at the pore corners. These waves created near each cavity mouth are coupled with the ones from the neighboring pores, and leads to oscillations in the flow field above the porous surface. As these acoustic disturbances travel back and forth between pores, the wavelength of the oscillations $\lambda_{\text {res }}$ is related to the spacing between cavities by $\lambda_{r e s}=s$. The nondimensionalized frequency of the resonant mode is then estimated as

$$
f_{\text {res }}=\frac{H}{\lambda_{\text {res }}}=\frac{\phi}{A r}
$$

Figure 12(a) shows a portion of the time-history of the tangential velocity $u$ at $\theta=10^{\circ}$ and $2 \mathrm{H}$ above a coating of porosity $\phi=0.2$, at $R e=100$ and 1000. At that location, the reflections of the acoustic pulse are measured earlier in time. Consequently, for $t \geq 20$, the resonant mode is the only unsteady feature of the flow. Similar oscillations, largely independent of location on the computational grid, are observed for the other flow field variables. As expected, the oscillation amplitude of the acoustic resonant modes decreases with $R e$ because of viscous absorption, and for lower Reynolds number (i.e., $R e=10$ ), the oscillations are completely damped. The corresponding power spectrum is shown in figure $12(b)$, and the predicted resonant frequency from Eq. 8 (represented by the vertical line) is in excellent agreement with the DNS measurement. We also observe a significant contribution from the first subharmonic of frequency $f_{\text {res }} / 2$ (i.e., of wavelength $2 s$ ) which corresponds to interaction between cavities one pore away from each other.

To quantify the relevance of the resonant mode compared to the other acoustic reflections, the power spectrum of the full time-signal without wall and from the reflection off the solid surface are also presented in figure 12(b). At the resonant frequency and subharmonic, the energy content of the solid wall reflection and the resonant mode are similar for $R e=1000$, and the resonant frequency are less energetic at $R e=100$ because of viscous absorption.

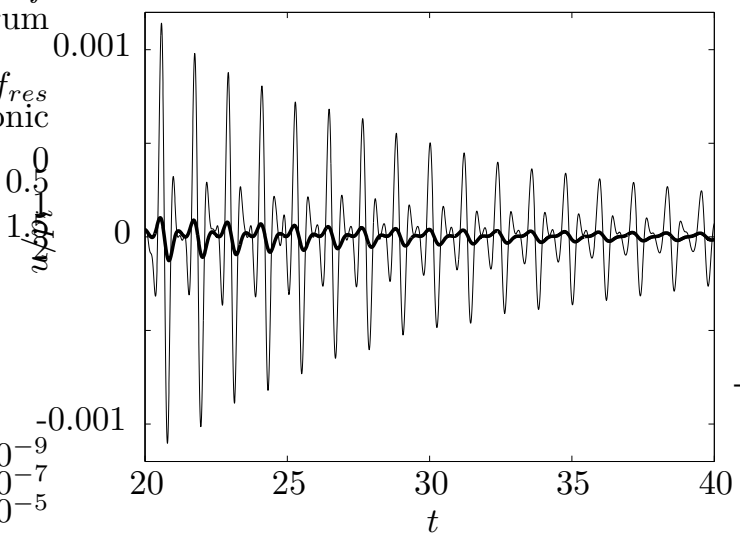

(a)

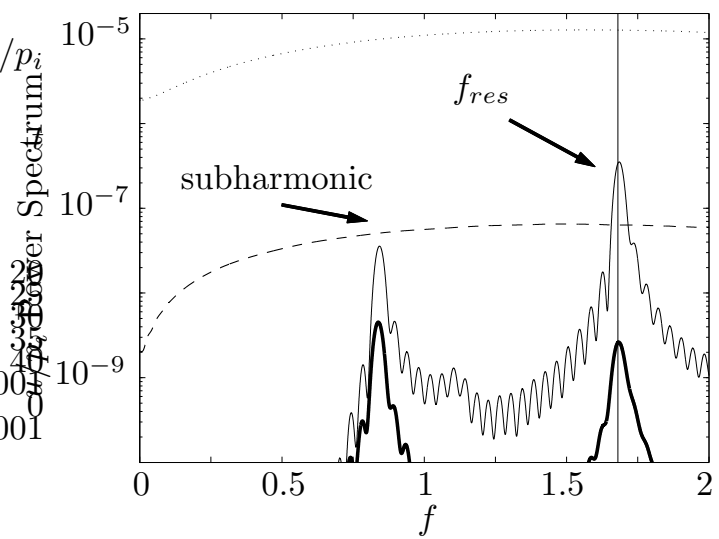

$(b)$

Figure 12. Details of the resonant mode for a UAC of porosity $\phi=0.2$ at $R e=100(-$ ) and $R e=$ 1000 ( - ). ( a) Velocity time-history for $t \geq 20$; (b) corresponding power spectrum. For comparison, the power spectrum of the full signal without wall $(\cdots \cdots \cdots)$ and from the reflection off the solid surface ( ----$)$ are also shown, for $R e=1000$. The vertical line correspond to the resonant frequency predicted with Eq. 8 . The results are shown for $\theta=10^{\circ}$ at $2 H$ above the wall.

Similar agreement between the measured and predicted resonant frequencies are obtained for porosity $\phi=0.48$ and 0.8 , with less than $2 \%$ error. In this mechanism, the resonant frequency depends on the cavity aspect ratio (constant in our study) and is proportional to porosity. Therefore, for coatings of high porosity, which are of interest for laminar-flow-control applications, these frequencies are higher than the ultrasonic 
frequency band relevant for UAC, and do not affect the computation of the reflection coefficients. However, the resonant disturbances may interact with the boundary-layer flow and cause detrimental tripping effect. This issue should be addressed in future work with the presence of the outer boundary-layer flow.

\section{E. Guidelines for UAC design}

For the 2-D micro-cavities considered in this study, the first important parameter is the cavity depth. In the range of acoustic Reynolds numbers relevant for practical UAC, reflections from the bottom of the cavities leads to minima of the reflection coefficient as some specific frequencies. Using the estimations from Eq. 6 or 7, the cavity depth $H$ could be chosen so that the predicted frequency of minimum reflection matches the frequency of the most amplified second-mode instability waves observed in experiments. Since our results show that this mechanism is only weakly affected by the porosity, the parameter $H$ could be tuned up independently of the parameter $\phi$. While the length-to-depth ratio of the pores was kept constant in our study, matching typical values from experiments, more simulations are underway to examine in details the effects of the cavity aspect ratio parameter.

Porosity is the other critical parameter for UAC design. Previous numerical simulations ${ }^{3}$ have suggested that UAC performance increases with porosity, and our parametric study tends to confirm this result. Overall, the amplitude of the reflection coefficient decreases with higher porosity, as the scattering and absorption of acoustic waves by the UAC is enhanced. Furthermore, for high porosity, the resonant acoustic mode has a frequency much higher than the ultrasonic frequency band relevant for UAC, and is not expected to significantly affect the performance. More work is required to investigate these acoustic modes, in particular for three-dimensional pores.

In theory, the most promising configurations correspond to square-rectangular and honeycomb 3-D patterns, with porosity up to 70-90\% (see Ref. 19). For such geometries, high-fidelity simulations are required to accurately describe the interactions between closely spaced pores. Additional work might also be needed to formulate and verify experimentally the structural integrity of these high-porosity coatings. Future studies will therefore focus on extending the current UAC research to realistic three-dimensional high-porosity coatings, with circular cavities, rectangular pores, or more complex geometry.

Finally, our DNS results show that the theoretical model presented in section IB is a robust and accurate tool for UAC design. The theoretical prediction of the reflection coefficient also agrees well with benchmark (no flow) measurement ${ }^{17}$ conducted for a UAC of regular microstructure at various ambient pressures, with emphasis on low pressures relevant to high-altitude hypersonic flights. This set of theoretical, numerical and experimental tools can be valuable to estimate in an economical way the acoustic properties of UAC samples before their testing in hypersonic wind tunnels.

\section{Summary}

The interaction of incident acoustic waves with an array of equally-spaced micro-cavities on a flat plate surface was investigated using theoretical modeling and direct numerical simulations. Since the second mode instability of hypersonic boundary layer represents trapped acoustic waves of ultrasonic frequency band, it is assumed that basic features of its interaction with porous coating can be captured by considering this acoustic scattering problem with no external flow.

The simulations were performed for a porous coating consisting of 2-D cavities of constant length to depth ratio, with an incoming circular acoustic pulse as initial condition. The reflection coefficient was computed as a function of the acoustic wave frequency and angle of incidence, for coatings of different porosity, at various Reynolds numbers relevant to hypersonic flight. Comparisons with theoretical prediction showed excellent agreement with the DNS results in the parametric range relevant to UAC applications for laminar flow control. Overall, the reflection off the UAC decreases with higher porosity, and in most cases, minima of the reflection coefficient exist at some specific frequencies. A model to predict these frequencies was proposed, and showed good agreement with the numerical and theoretical data.

The simulations also highlighted the presence near the UAC surface of resonant acoustic modes caused by the coupling of small-scale scattered waves generated by neighboring pores. A formulation to estimate the resonant frequency was presented, and the predicted frequencies agreed well with DNS measurements. While the resonant modes were not captured by the theoretical model, they are mainly relevant for coatings with cavity spacing of the same order than the wavelength of the incident wave. In practice, typical UAC for 
laminar-flow-control applications has spacing less than 0.1 of the second-mode wavelength instability; i.e., the resonant frequency is much larger than the second-mode frequency. Nevertheless, the resonant acoustic modes may be excited by high-frequency disturbances of the outer flow and trip the boundary layer similar to small-scale distributed roughness. Feasibility of this detrimental effect should be addressed in future numerical simulations including the outer flow.

Finally, based on our parametric study of the geometrical factors and flow conditions effects, we identified the cavity depth $H$ and the porosity $\phi$ as the most important parameters for UAC design. Guidelines for the choice of these parameters were also suggested. It is our hope that a better understanding of these acoustic properties will lead to improvements in existing UAC models.

\section{Acknowledgments}

The authors would like to acknowledge contributions to this work by Dr. Norm Malmuth, whose passionate work on diverse topics, including UAC, and generosity to his collaborators will be sorely missed. This work was supported by the Air Force of Scientific Research under Contract FA9550-06-C-0097.

\section{References}

${ }^{1}$ Malik, M. R., Zang, T. A., and Bushnell, D. M., "Boundary layer transition in hypersonic flows," AIAA Paper 90-5232, 1990.

${ }^{2}$ Kimmel, R., "Aspects of hypersonic boundary layer transistion control," AIAA Paper 2003-0772, 2003.

${ }^{3}$ Fedorov, A. V., Malmuth, N. D., Rasheed, A., and Hornung, H. G., "Stabilization of hypersonic boundary layers by porous coatings," AIAA J., Vol. 39(4), 2001, pp. 605-610.

${ }^{4}$ Rasheed, A., Hornung, H. G., Fedorov, A. V., and Malmuth, N. D., "Experiments on passive hypervelocity boundary layer control using an ultrasonically absorptive surface," AIAA J., Vol. 40(3), 2002, pp. 481-489.

${ }^{5}$ Fedorov, A. V., Shiplyuk, A. N., Maslov, A. A., Burov, E. V., and Malmuth, N. D., "Stabilization of a hypersonic boundary layer using and ultrasonically absorptive coating," J. Fluid Mech., Vol. 479, 2003, pp. 99-124.

${ }^{6}$ Fedorov, A. V., Kozlov, V. F., Shiplyuk, A. N., Maslov, A. A., Sidorenko, A. A., Burov, E. V., and Malmuth, N. D., "Stability of hypersonic boundary layer on porous wall with regular microstructure," AIAA Paper 2003-4147, 2003.

${ }^{7}$ Bountin, D., Shiplyuk, A., Maslov, A., and Chokani, N., "Nonlinear aspects of hypersonic boundary layer stability on a porous surface," AIAA Paper 2004-0255, 2004.

${ }^{8}$ Maslov, A. A., Shiplyuk, A. N., Sidorenko, A. A., Polivanov, P., Fedorov, A. V., Kozlov, V. F., and Malmuth, N. D., "Hypersonic laminar flow control using a porous coating of random microstructure," AIAA Paper 2006-1112, 2006.

${ }^{9}$ Kinsler, L. E., Frey, A. R., Coppens, A. B., and Sanders, J. V., Fundamentals of acoustics, John Wiley \& Sons Inc., New York, 4th ed., 2000.

${ }^{10}$ Lependin, L. F., Acoustics, Moscow, Vysshaya Shkola, 1978.

${ }^{11}$ Kozlov, V. F., Fedorov, A. V., and Malmuth, N. D., "Acoustic properties of rarefied gases insides pores of simple geometries," J. Acoust. Soc. Amer., Vol. 117(6), 2005, pp. 3402-3412.

${ }^{12}$ Brès, G. A. and Colonius, T., "Three-dimensional instabilities in compressible flow over open cavities," J. Fluid Mech., Vol. 599, 2008, pp. 309-339.

${ }^{13}$ Pierce, A. D., Acoustics, an introduction to its physical principles and applications, McGraw-Hill, 1989.

${ }^{14}$ Stetson, K. F., Thomson, E. R., Donaldson, J. C., and Siler, L. G., "Laminar boundary-layer stability experiments on a cone at Mach 8, part 1: sharp cone," AIAA Paper 83-1761, 1983.

${ }^{15}$ Egorov, I. V., Fedorov, A. V., Novikov, A. V., and Soudakov, V. G., "Direct numerical simulation of supersonic boundarylayer stabilization by porous coatings," AIAA Paper 2007-948, 2007.

${ }^{16}$ Brès, G. A., Colonius, T., and Fedorov, A. V., "Stability of temporally evolving supersonic boundary layers over microcavities for ultrasonic absorptive coatings," AIAA Paper 2008-4337, 2008.

${ }^{17}$ Fedorov, A. V., Kozlov, V. F., and Addison, R. C., "Reflection of acoustic disturbances from a porous coating of regular microstructure," AIAA Paper 2008-3902, 2008.

${ }^{18}$ Malmuth, N. D., Fedorov, A. V., Shalaev, V., Cole, J., Khokhlov, A., Hites, M., and Williams, D., "Problems in high speed flow prediction relevant to control," AIAA Paper 98-2695, 1998.

${ }^{19}$ Fedorov, A. V. and Malmuth, N. D., "Parametric studies of hypersonic laminar flow control using a porous coating of regular microstructure," AIAA Paper 2008-588, 2008. 